\title{
ON THE NON-LINEAR VIBRATION OF ELASTIC BARS*
}

\author{
BY \\ A. CEMAL ERINGEN \\ Illinois Institute of Technology
}

1. Introduction. The classical theory of vibration of bars is based on certain restrictive assumptions, namely: (a) the deflection is small; (b) supports are free to move in the axial direction; (c) deflection is inextensional. In practice, however, very often some or all of these assumptions are violated. Therefore, it is necessary to reformulate the problem of vibration of bars in its general form without these assumptions so that the domain of applicability of the classical theory can be well defined and problems to which the classical theory is not applicable can be attacked.

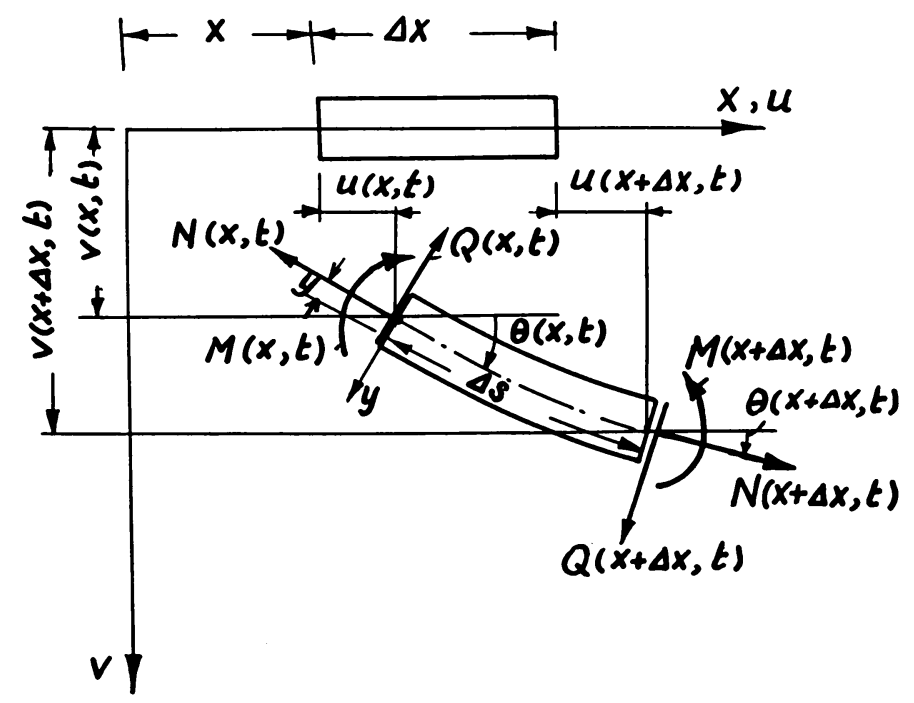

FIG. 1. Displaced element of beam.

Recently Woinowsky-Krieger [1] studied the effect of axial force on the vibration of hinged bars. N. J. Hoff [2] gave an analysis for the effect of inertia forces on the buckling of columns. In both of these analyses the classical treatment was improved by consideration of the axial stress due to bending.

In the following analysis, first the problem of vibration of bars is reformulated without any of the above-mentioned assumptions. Following that, a basic problem, free vibration of elastic bars having immovable hinged ends, is solved with the use of the perturbation method. The solution of this problem adequately describes those motions in which the changes in axial tension, as well as in deflection, are not small.

Solutions of vibration problems concerning bars with other types of end conditions and forced vibrations are left to a forthcoming paper.

*Received Feb. 15, 1951. 
2. Equations of motion. The equations of dynamic equilibrium of an element of the beam deformed to a plane figure are (Fig. 1)

$$
\begin{gathered}
-\rho A u_{. t t}+(N \cos \theta)_{. x}-(Q \sin \theta)_{. x}=0, \\
-\rho A v_{, t t}+(N \sin \theta)_{. x}+(Q \cos \theta)_{. x}=0, \\
\left(1+u_{, x}\right) J \theta_{. t t}+M_{, x}-Q\left[\left(1+u_{. x}\right) \cos \theta+v_{, x} \sin \theta\right] \\
+N\left[v_{. x} \cos \theta-\left(1+u_{, x}\right) \sin \theta\right]=0 .
\end{gathered}
$$

where $\rho$ is the mass per unit volume, $A$ the cross-sectional area of the beam, $J$ the mass moment inertia per unit length, $N, Q, M$ are the axial force, the shear force, and the bending moment at a point $x, u$ and $v$ are the deflections of any point $x$ in the axial and transverse directions, $\theta$ is the angle between the tangent to the median line and the $x$ axis, and $t$ is the time. In calculating the inertia forces, the effect of changes of mass during motion is neglected.

The length element, $d s$, after deformation is given by

$d s^{2}=\left\{\left[v_{. x}+(y \cos \theta)_{. x}\right]^{2}+\left[1+u_{, x}-(y \sin \theta)_{, x}\right]^{2}\right\} d x^{2}, \quad \tan \theta=v_{, x} /\left(1+u_{, x}\right)$.

Indices after a comma represent differentiation. In deriving Eq. (2) the effect of shear deformation is neglected.

Normal strain is defined as

$$
e=\frac{d s}{d x}-1
$$

Equations (2) and (3) are combined to yield

$$
e=\epsilon-y \theta_{, x}, \quad \epsilon=\frac{1+u_{. x}}{\cos \theta}-1=\frac{v_{. x}}{\sin \theta}-1 .
$$

Here, $\epsilon$ is the strain referred to the median line of the undeformed beam.

Hooke's Law states that

$$
\sigma=E e .
$$

Thus,

$$
N=\int \sigma d A=E A \epsilon, \quad M=\int \sigma y d A=-E I \theta_{, x}
$$

are calculated with the use of the first Eq. (4). Here $I$ is the moment inertia of any section about its neutral axis. The variation of $I$ with time is neglected.

Equations (6) and the third Eq. (1) are combined to give

$$
Q=-\frac{\left(E I \theta_{. x}\right)_{. x}}{1+\epsilon}+J \theta_{. t t} \cos \theta .
$$

The first two Eqs. (1) can be transformed into more suitable form by introducing non-dimensional quantities and multiplying the second by $i=\sqrt{-1}$ and adding the result to the first. The resulting equation is differentiated with respect to $x$. Thus, 


$$
\begin{gathered}
-\left[(1+\epsilon) e^{i \theta}\right]_{, \tau \tau}+\left\{\left[\epsilon+i \lambda^{2}\left(-\frac{\theta_{. \nu \nu}}{1+\epsilon}+\theta_{, \tau \tau} \cos \theta\right)\right] e^{i \theta}\right\}_{, \nu \nu}=0 \\
\tau=\left(E / \rho L^{2}\right)^{1 / 2} t, \quad y=x / L, \quad \lambda^{2}=J / \rho A L^{2} .
\end{gathered}
$$

The second Eq. (4) may be transformed to

$$
y+\frac{1}{L}[u(x, t)+i v(x, t)-u(0, t)-i v(0, t)]=\int_{0}^{y}(1+\epsilon) e^{i \theta} d y .
$$

The first bracket in the first Eq. (8) represents the effect of translational inertia; the first, second, and third terms in the brace are respectively the contributions of the extension of the median line, the bending, and the rotatory inertia.

For $\lambda=0$ the first Eq. (8) reduces to the one obtained by Carrier [4] for the problem of non-linear vibration of the elastic string, when $\epsilon$ is replaced by $\left(\epsilon-\epsilon_{0}\right)$ in order to include the initial tension.

Further, let

$$
s=\lambda \tau=t\left(E I g / W L^{3}\right)^{1 / 2},
$$

where $W$ is the total weight of the beam. Equations (8), when separated into real and imaginary parts, become

$$
\begin{aligned}
& -\lambda^{2} \epsilon_{, s s}+(1+\epsilon) \lambda^{2} \theta_{, s}^{2}+\epsilon_{, y y}-\epsilon \theta_{, y}^{2}+\frac{2 \lambda^{2} \theta_{, y} \theta_{, y y y}}{1+\epsilon}-2 \lambda^{2} \frac{\epsilon_{, y} \theta_{, y} \theta_{, y y}}{(1+\epsilon)^{2}} \\
& +\lambda^{2} \frac{\theta_{, \nu \nu}^{2}}{1+\epsilon}-2 \lambda^{4} \theta_{, s s y} \theta_{, \nu} \cos \theta+2 \lambda^{4} \theta_{, s s} \theta_{, \nu}^{2} \sin \theta-\lambda^{4} \theta_{, s s} \theta_{, y \nu} \cos \theta=0, \\
& -2 \lambda^{2} \epsilon_{, s} \theta_{, s}-(1+\epsilon) \lambda^{2} \theta_{, s s}+2 \epsilon_{, y} \theta_{, y}+\epsilon \theta_{, y \nu}-\lambda^{2} \frac{\theta_{, y y y \nu}}{1+\epsilon}+2 \lambda^{2} \frac{\epsilon_{, \nu} \theta_{. \nu y y}}{(1+\epsilon)^{2}} \\
& +\lambda^{2} \frac{\epsilon_{. y \nu} \theta_{. \nu y}}{(1+\epsilon)^{2}}-2 \lambda^{2} \frac{\epsilon_{. \nu}^{2} \theta_{. \nu \nu}}{(1+\epsilon)^{3}}+\lambda^{2} \frac{\theta_{. \nu}^{2} \theta_{, y y}}{1+\epsilon}+\lambda^{4} \theta_{, s s y y} \cos \theta-2 \lambda^{4} \theta_{, y} \theta_{, s s y} \sin \theta \\
& -2 \lambda^{4} \theta_{, y}^{2} \theta_{, s s} \cos \theta-\lambda^{4} \theta_{, s s} \theta_{, y \nu} \sin \theta=0 .
\end{aligned}
$$

Contrary to expectation, it can be seen that $\epsilon=0$ is not a possible solution since Eqs. (11) and (12) reduce to two independent equations for $\theta$. Consequently, assumption (c) of the classical theory is not valid. It can be seen, however, that this assumption will be valid when $\epsilon$ is a higher-order quantity than $\theta$. Therefore, the classical theory is obtained as a limiting case of the present theory for $\theta \rightarrow 0$.

3. Beams with immovable hinged ends. The boundary conditions for simplysupported beams with immovable hinged ends are

$$
\theta_{, y}=0, \quad u=v=0 \quad \text { for } y=0,1 \text {. }
$$

The perturbation parameter is chosen as $\lambda$ which represents the ratio of rotational inertia to translational inertia. Reversal of the $\operatorname{sign}$ of $\lambda$ should reverse the sign of $\theta$ but not $\epsilon ; \theta$ and $\epsilon$ are therefore expanded into power series of $\lambda$ as follows

$$
\theta=\lambda \theta_{1}+\lambda^{3} \theta_{3}+\lambda^{5} \theta_{5}+\cdots, \quad \epsilon=\lambda^{2} \epsilon_{2}+\lambda^{4} \epsilon_{4}+\cdots .
$$


The expressions for $\theta$ and $\epsilon$ are substituted into Eqs. (11) and (12) to obtain differential equations and into (13) and (9) to obtain boundary conditions.

Differential equations

$\left(\lambda^{2}\right): \quad \epsilon_{2, \nu \nu}=0$,

$\left(\lambda^{4}\right): \quad \epsilon_{4, y y}=\epsilon_{2, s s}-\theta_{1, s}^{2}-2 \theta_{1, y} \theta_{1, y \nu \nu}-\theta_{1, y \nu}^{2}-2 \epsilon_{2} \epsilon_{2, y \nu}+\epsilon_{2} \theta_{1, \nu}^{2}$,

$\left(\lambda^{6}\right): \quad \cdots$,

$\left(\lambda^{3}\right): \quad \theta_{1, s s}+\theta_{1, y y y \nu}-\epsilon_{2} \theta_{1, y \nu}-2 \epsilon_{2, y} \theta_{1, y}=0$,

$\left(\lambda^{5}\right): \quad \theta_{3, s s}+\theta_{3, v y v y}-\epsilon_{2} \theta_{3, v y}=\theta_{1, s s y v}-2 \epsilon_{2, s} \theta_{1, s}-4 \epsilon_{2} \theta_{1, s s}$

$$
\begin{aligned}
& -2 \epsilon_{2} \theta_{1, y \nu y y}+2 \epsilon_{2, y} \theta_{1, y y y}+\epsilon_{2, y y} \theta_{1, y y}+\theta_{1, y}^{2} \theta_{1, y \nu}+2 \epsilon_{2, y} \theta_{3, \nu} \\
& +2 \epsilon_{4, \nu} \theta_{1, \nu}+6 \epsilon_{2} \epsilon_{2, y} \theta_{1, y}+\epsilon_{4} \theta_{1, y \nu}+3 \epsilon_{2}^{2} \theta_{1, y \nu}=0,
\end{aligned}
$$

$\left(\lambda^{7}\right): \quad \cdots ;$

Boundary conditions

at

$$
y=0,1: \quad \theta_{i, \nu}=0, \quad(i=1,2, \cdots)
$$

$$
\begin{array}{lc}
(\lambda): \int_{0}^{1} \theta_{1} d y=0, & \left(\lambda^{2}\right): \int_{0}^{1}\left(\epsilon_{2}-\frac{\theta_{1}^{2}}{2}\right) d y=0, \\
\left(\lambda^{3}\right): \int_{0}^{1}\left(\epsilon_{2} \theta_{1}+\theta_{3}-\frac{1}{6} \theta_{1}^{3}\right) d y=0,\left(\lambda^{4}\right): \int_{0}^{1}\left(\epsilon_{4}-\epsilon_{2} \frac{\theta_{1}^{2}}{2}-\theta_{1} \theta_{3}+\frac{\theta_{1}^{4}}{24}\right) d y=0, \\
\left(\lambda^{5}\right): \cdots, & (18),\left(\lambda^{6}\right): \cdots ;
\end{array}
$$

Initial conditions

$$
\begin{aligned}
& \text { at } \quad s=0: \quad \theta_{1}=m \pi \theta_{0} \cos m \pi y, \quad \theta_{i}=0, \quad \theta_{i, s}=0, \quad \epsilon_{i, s}=0, \\
& (i=1,2, \cdots ; j \neq 1)
\end{aligned}
$$

The solution of the first Eq. (15) under the condition that $\epsilon_{2}(0, s)=\epsilon_{2}(1, s)$ and the first Eq. (19) is

$$
\epsilon_{2}=\frac{1}{2} \int_{0}^{1} \theta_{1}^{2} d y
$$

Substitution of (21) into the first Eq. (16) leads to

$$
\theta_{1, s s}+\theta_{1, y y y y}-\frac{1}{2} \theta_{1, y y} \int_{0}^{1} \theta_{1}^{2} d y=0 .
$$

Equation (22) is solved under the boundary conditions (17) and the first Eq. (18) for an initial sinusoidal deflection given by the first Eq. (20).

Let

$$
\theta_{1}=m \pi \theta_{0} \cdot S(s) \cos m \pi y, \quad S(0)=1 .
$$


Substitution of Eq. (23) into Eq. (22) gives the following differential equation:

$$
S_{, s s}+(m \pi)^{4} S+\frac{1}{4}(m \pi)^{4} \theta_{0}^{2} S^{3}=0 .
$$

Multiplying (24) by $S_{, s}$, a first integral can be obtained immediately. The resulting equation is of first order and separable. Integration can be effected in terms of elliptic functions. The result is

$$
\begin{array}{ll}
\theta_{1}=m \pi \theta_{0} \cos m \pi y \text { cn }\left(\omega_{1} s, k\right), & \omega_{1}=m^{2} \pi^{2}\left(1+\frac{1}{4} \theta_{0}^{2}\right)^{1 / 2}, \\
k^{2}=\left(2+\frac{8}{\theta_{0}^{2}}\right)^{-1}, & \frac{T_{1}}{T_{0}}=\frac{2}{\pi}\left(1+\frac{\theta_{0}^{2}}{4}\right)^{-1 / 2} K,
\end{array}
$$

where cn and $K$ denote the elliptic cosine and the complete elliptic integral of the first kind; $T_{1} / T_{0}$ is the ratio of non-linear period to linear period. The use of Eq. (21) gives

$$
\epsilon_{2}=\frac{1}{4}\left(m \pi \theta_{0}\right)^{2} \mathrm{cn}^{2}\left(\omega_{1} s, k\right) .
$$

Substitution of (25) and (26) into the second Eq. (15), integration, and the condition $\epsilon_{4}(0, s)=\epsilon_{4}(1, s)$, yield

$$
\begin{aligned}
\epsilon_{4} & =e_{2}(s) \cos 2 m \pi y+e_{4}(s), \\
e_{2}(s) & =\frac{(m \pi)^{4}}{8}\left[\theta_{0}^{2}+\frac{1}{8} \theta_{0}^{4}+2 \theta_{0}^{2} \mathrm{cn}^{2}\left(\omega_{1} s, k\right)+\frac{1}{8} \theta_{0}^{4} \mathrm{cn}^{4}\left(\omega_{1} s, k\right)\right], \\
e_{4}(s) & =\frac{3}{64}\left(m \pi \theta_{0}\right)^{4} \mathrm{cn}^{4}\left(\omega_{1} s, k\right)+m \pi \theta_{0} \operatorname{cn}\left(\omega_{1} s, k\right) \int_{0}^{1} \theta_{3} \cos m \pi y d y,
\end{aligned}
$$

where $e_{4}(s)$ is obtained after the use of the second Eq. (19). The second Eq. (16) thus becomes

$$
\begin{aligned}
& \theta_{3, s s}+\theta_{3, y y y y}-\frac{1}{4}\left(m \pi \theta_{0}\right)^{2} \mathrm{cn}^{2}\left(\omega_{1} s, k\right) \theta_{3, y y}+(m \pi)^{4} \theta_{0}^{2} \mathrm{cn}^{2}\left(\omega_{1} s, k\right) . \\
& \cos m \pi y \int_{0}^{1} \theta_{3} \cos m \pi y d y=R_{1}(s) \cos m \pi y+R_{2}(s) \cos 3 m \pi y, \\
& \begin{array}{r}
R_{1}(s)=(m \pi)^{7}\left[\left(1-\frac{13}{16} \theta_{0}^{2}-\frac{13}{128} \theta_{0}^{4}\right) \theta_{0} \operatorname{cn}\left(\omega_{1} s, k\right)+\frac{15}{8} \theta_{0}^{3} \mathrm{cn}^{3}\left(\omega_{1} s, k\right)\right. \\
\left.+\frac{21}{128} \theta_{0}^{5} \mathrm{cn}^{5}\left(\omega_{1} s, k\right)\right], \\
R_{2}(s)=(m \pi)^{7}\left[-\frac{5}{16} \theta_{0}^{3} \mathrm{cn}\left(\omega_{1} s, k\right)-\frac{5}{128} \theta_{0}^{5} \mathrm{cn}\left(\omega_{1} s, k\right)-\frac{3}{8} \theta_{0}^{3} \mathrm{cn}^{3}\left(\omega_{1} s, k\right)\right. \\
\left.\quad-\frac{5}{128} \theta_{0}^{5} \mathrm{cn}^{5}\left(\omega_{1} s, k\right)\right] .
\end{array}
\end{aligned}
$$


The solution of partial differential equation (28) is of the following form:

$$
\theta_{3}=\theta_{3}^{(0)}+\theta_{3}^{(1)}+\theta_{3}^{(2)} \text {. }
$$

where $\theta_{3}^{(0)}$ is the solution of the reduced equation. $\theta_{3}^{(1)}$ and $\theta_{3}^{(2)}$ are particular solutions of the differential equations obtained by taking $R_{2}(s) \equiv 0$ and $R_{1}(s) \equiv 0$ respectively. An examination of the second Eq. (18) reveals that

$$
\theta_{3}^{(0)}=S_{1}(\sigma) \cos m \pi y, \quad \theta_{3}^{(0)}=S_{2}(\sigma) \cos r \pi y, \quad \sigma=\omega_{1} s, \quad r \neq m,
$$

where $S_{1}$ and $S_{2}$ satisfy the following differential equations which are obtained by substituting Eqs. (30) into the reduced equation

$$
\begin{aligned}
& S_{1, \sigma \sigma}=\left[2 k^{2} \operatorname{sn}^{2}(\sigma, k)-1\right] S_{1}, \\
& S_{2, \sigma \sigma}=\left[6 k^{2} \operatorname{sn}^{2}(\sigma, k)-\left(1+4 k^{2}\right)\right] S_{2} .
\end{aligned}
$$

Differential equations (31) and (32) are the Jacobian forms of the generalized Lamé equation. The solutions of these equations are:

$$
\begin{gathered}
S_{\alpha}=B \Lambda_{\alpha}^{(1)}+C \Lambda_{\alpha}^{(2)}, \quad(\alpha=1,2) \\
\Lambda_{1}^{(1)}=\operatorname{cn}(\sigma, k), \\
\Lambda_{1}^{(2)}=\operatorname{cn}(\sigma, k)\left[\left(1-k^{2}\right) \sigma+\operatorname{dn}(\sigma, k) \operatorname{sc}(\sigma, k)-E(\sigma, k)\right] ; \\
\Lambda_{2}^{(1)}=\prod_{r=1}^{2} \frac{H\left(\sigma+\sigma_{r}\right)}{\theta(\sigma)} \exp \left[-\sigma Z\left(\sigma_{r}\right)\right], \\
\Lambda_{2}^{(2)}=\prod_{r=1}^{2} \frac{H\left(\sigma-\dot{\sigma}_{r}\right)}{\theta(\sigma)} \exp \left[\sigma Z\left(\sigma_{r}\right)\right],
\end{gathered}
$$

where $\sigma_{1}$ and $\sigma_{2}$ are chosen to satisfy the following two independent equations:

$$
\begin{gathered}
\operatorname{sn} \sigma_{1} \text { en } \sigma_{1} \operatorname{dn} \sigma_{1}+\operatorname{sn} \sigma_{2} \text { en } \sigma_{2} \operatorname{dn} \sigma_{2}=0, \\
\left(\operatorname{cn} \sigma_{1} \operatorname{ds} \sigma_{1}+\operatorname{cn} \sigma_{2} \operatorname{ds~} \sigma_{2}\right)^{2}-\mathrm{ns}^{2} \sigma_{1}-\mathrm{ns}^{2} \sigma_{2}=-\left(1+4 k^{2}\right) .
\end{gathered}
$$

Here $\Theta(u), H(u)$, and $Z(u)$ are Jacobian Theta, Eta and Zeta functions respectively [4]; $E(u)$ is the fundamental elliptic integral of the second kind; sn $u$, cn $u$, and dn $u$ are Jacobian elliptic functions and sc $u=\operatorname{sn} u / \operatorname{cn} u, \operatorname{ds} u=\operatorname{dn} u / \operatorname{sn} u$, ns $u=1 / \operatorname{sn} u$ as originated by Glaisher. These functions are tabulated in [6].

The general solution of an equation similar to (32) was first given by Hermite [5]. Only the first of Eqs. (34) can be extracted from this solution by use of properties of these functions. The second solution $\Lambda_{1}^{(2)}$ is obtained upon reducing the order of the differential Eq. (31) by letting $\Lambda_{1}^{(2)}=\Lambda(\sigma)$ cn $(\sigma, k)$ and upon integrating the resulting equation for $\Lambda(\sigma)$. It can be seen that while $\Lambda_{1}^{(1)}$ is a doubly-periodic function $\Lambda_{1}^{(2)}$ is not periodic.

Particular solutions $\theta_{3}^{(1)}$ and $\theta_{3}^{(2)}$ of Eqs. (28) are of the following forms:

$$
\theta_{3}^{(1)}=S^{(1)}(\sigma) \cos m \pi y, \quad \theta_{3}^{(2)}=S^{(2)}(\sigma) \cos 3 m \pi y .
$$


Substitution of Eqs. (37) into the first of Eqs. (28) with $R_{2}(s) \equiv 0$ and $R_{1}(s) \equiv 0$ respectively, results in

$$
\begin{aligned}
& S_{, \sigma \sigma}^{(1)}-\left[2 k^{2} \operatorname{sn}^{2}(\sigma, k)-1\right] S^{(1)}=R_{1}(\sigma), \\
& S_{, \sigma \sigma}^{(2)}-\left[6 k^{2} \operatorname{sn}^{2}(\sigma, k)-\left(1+4 k^{2}\right)\right] S^{(2)}=R_{2}(\sigma) .
\end{aligned}
$$

Particular solutions of these equations are obtained by Lagrange's method of variation of parameters. Hence*

$$
\begin{aligned}
S^{(\alpha)}(\sigma) & =\frac{1}{\Delta_{\alpha}} \int_{0}^{\sigma}\left[\Lambda_{\alpha}^{(1)}(\sigma) \cdot \Lambda_{\alpha}^{(2)}(t)-\Lambda_{\alpha}^{(2)}(\sigma) \Lambda_{\alpha}^{(1)}(t)\right] R_{\alpha}(t) d t \\
\Delta_{1} & =-\left(1-k^{2}\right), \quad(\alpha=1,2) \\
\Delta_{2} & =2 \frac{\prod_{i=1}^{2} H\left(\sigma_{i}\right) H\left(-\sigma_{i}\right)}{\theta^{2}(0)} \sum_{i=1}^{2} \operatorname{cn} \sigma_{i} \operatorname{dn} \sigma_{i} \operatorname{ns} \sigma_{i},
\end{aligned}
$$

where $\sigma_{1}$ and $\sigma_{2}$ must be solved from Eq. (36). Finally, the use of the initial conditions given by Eq. (20), namely, $\theta_{3}=\theta_{3, s}=0$ for $s=0$ reduce the general solution $\theta_{3}(y, \sigma)$ to:

$$
\theta_{3}(y, \sigma)=S^{(1)}(\sigma) \cos m \pi y+S^{(2)}(\sigma) \cos 3 m \pi y .
$$

$e_{4}(s)$ of Eq. (27) thus becomes

$$
e_{4}(s)=\frac{3}{64}\left(m \pi \theta_{0}\right)^{4} \mathrm{cn}^{4}\left(\omega_{1} s, k\right)+\frac{1}{2} m \pi \theta_{0} S^{(1)}\left(\omega_{1} s\right) \text { cn }\left(\omega_{1} s, k\right)
$$

This completes the solution of the equations corresponding to $\lambda^{4}$ and $\lambda^{5}$. Further approximation requires more tedious analysis. However, since larger initial deflection will cause extremely high axial tension in the bar, the validity of Hooke's Law must be examined before taking any further steps toward improvement.

There still remains the examination of the question of convergence. This is impossible at this point. Although a term, $\sigma$, appears in the expression for $\Lambda_{1}^{(2)}$, this does not necessarily mean a resonance effect, since further approximations are necessary to examine the series in $\sigma$ for the question of stability.

An estimate of region of stability of Eqs. (31) and (32) can be made for small $k$. In this case $\sin \sigma$ can be used in place of sn $\sigma$. Therefore Eqs. (31) and (32) become Mathieu equations whose stability regions in terms of parameter $k^{2}$ are well known [7], and can easily be seen to contain those of the present equations.

4. Vibration produced by an arbitrary initial deflection. Analysis of the preceding section is based on an initial condition corresponding to an initial sinuzoidal deflection of the hinged bar. Differential equations must be re-solved when the initial deflection is arbitrarily prescribed, since these equations are non-linear. The problem of non-linear vibrations of the elastic string following an initial deflection has its analogue in the case of hinged elastic bars. Following a similar method, as that of [3] an integral equation is obtained below which may be solved by the method of successive approximations. This

*Eq. (40) for $\alpha=1$, can be integrated in closed form, in terms of elliptic functions. This result will not be given here, however. 
equation represents the $\lambda^{3}$-approximation of the problem since it is obtained by integrating Eq. (22). Let

$$
\begin{aligned}
\theta_{1}(y, s) & =\sum_{m} A_{m} S_{m}(s) \cos m \pi y, \\
\theta_{1}(y, 0) & =\sum_{m} A_{m} \cos m \pi y, \quad S_{m}(0)=1,
\end{aligned}
$$

where $A_{m}$ are the Fourier coefficients for the initial deflection function $\theta_{1}(y, 0)$. Substitution of Eq. (45) into Eq. (22) leads to

$$
S_{m, 8 s}+(m \pi)^{4} S_{m}=-\frac{(m \pi)^{2}}{4} S_{m} \sum_{n} A_{n}^{2} S_{n}^{2} .
$$

The required result is obtained by integrating Eq. (46):

$$
S_{m}(s)=\cos m^{2} \pi^{2} s-\frac{1}{4} \int_{0}^{s} \sin m^{2} \pi^{2}(s-t) S_{m}(t) \sum_{n} A_{n}^{2} S_{n}^{2}(t) d t .
$$

There remains the problem of solving $\epsilon_{4}, \epsilon_{6}, \cdots ; \theta_{3}, \theta_{5}$, etc., for an arbitrary initial deflection. However, corresponding differential equations given by (15) and (16) are linear. Therefore superposition is valid after $\theta_{1}(y, s)$ is solved from non-linear integral Eq. (47).

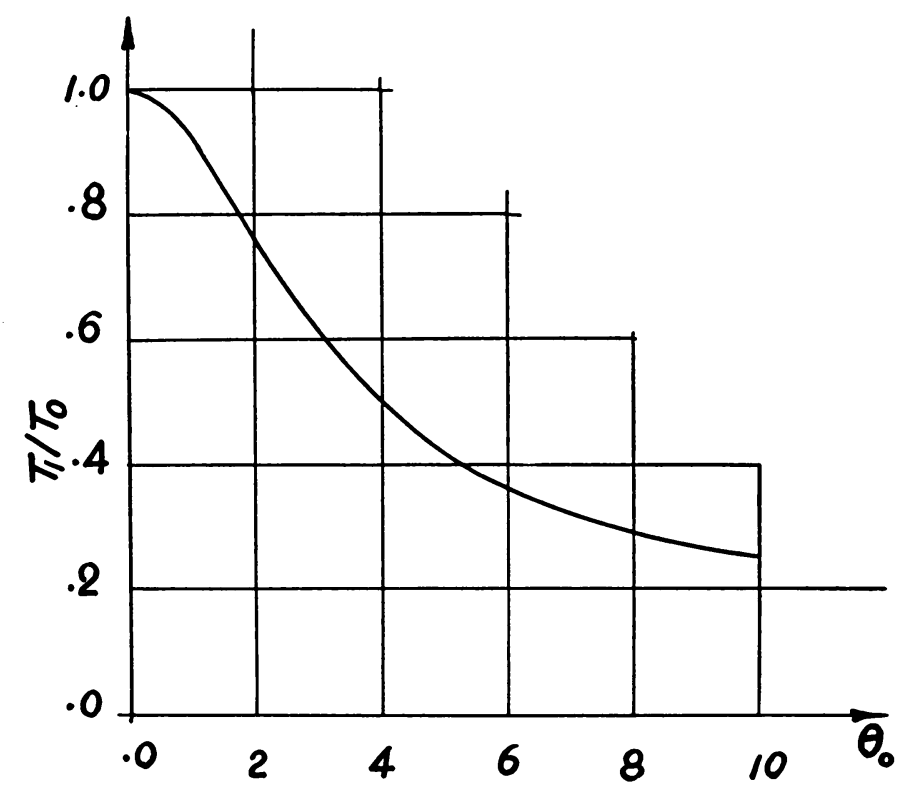

Frg. 2. Period versus initial deflection.

On Fig. 2 the ratio of non-linear period over linear period, $T_{1} / T_{0}$, is plotted against initial deflection angle $\theta_{0}$. It is seen that classical theory is correct only for vanishing $\theta_{0}$. Fig. (3) represents the axial stress multiplied by a scale $\left(1 / m^{2} \pi^{2} E\right)$ against $T_{1} / T_{0}$. 


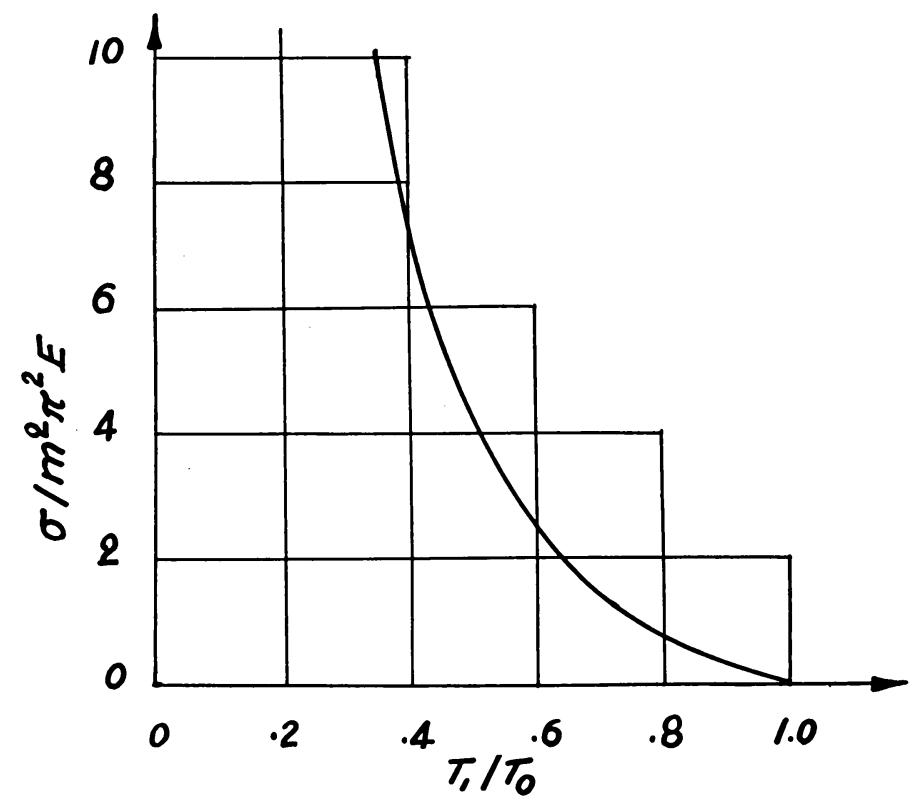

Fig. 3. Axial stress versus period.

It is seen that axial stress increases very rapidly with the decrease of $T_{1} / T_{0}$ or with the increase of frequency ratios.

\section{REFERENCES}

1. S. Woinowsky-Krieger, The effect of an axial force on the vibrations of hinged bars. J. Appl. Mech. 17, 35-36 (1950).

2. N. J. Hoff, The dynamics of the buckling of elastic columns. Paper presented at the 16th Meeting of the American Society of Mechanical Engineers, June 22-24, 1950.

3. G. F. Carrier, On the non-linear vibration problem of the elastic string, Q. Appl. Math. 3, 157-165 (1945).

4. G. F. Carrier, A note on the vibrating string, Q. Appl. Math. 7, 97-101 (1949).

5. E. T. Wittaker and G. N. Watson, A course of modern analysis, Cambridge University Press, 1944, pp. $479-480$, p. 573.

6. Edwin P. Adams and R. L. Hippisley, Smithsonian mathematical formulae and tables of elliptic functions, Smithsonian Institution, 1947.

7. J. J. Stoker, Non-linear vibrations, Interscience Publishers, 1950, p. 205. 